\title{
Transition from open to robotic-assisted radical prostatectomy: 7 years experience at Hackensack University Medical Center
}

\author{
Ravi Munver · Ilya A. Volfson · Stuart S. Kesler • \\ Michael Nazmy $\cdot$ Ihor S. Sawczuk
}

Received: 7 November 2006/ Accepted: 21 March 2007/Published online: 10 May 2007

(C) Springer London 2007

\begin{abstract}
Background and objective Open radical prostatectomy (ORP) is the standard approach for the surgical management of localized prostate cancer. The steep learning curve for laparoscopic prostatectomy poses a challenge for surgeons with minimal laparoscopic experience. As robotic-assisted surgery becomes more prevalent in the urologic community, there appears to be an increasing interest in robotic-assisted radical prostatectomy (RARP) among urologists throughout the United States. We report on the impact of robotics on practice patterns in the treatment of localized prostate cancer at a single institution.

Methods A retrospective review was conducted of radical prostatectomies performed between January 2000 and December 2006 at Hackensack University Medical Center (Hackensack, N.J.). Over this time period, our medical center acquired four da Vinci ${ }^{\mathrm{TM}}$ Surgical Systems. The trends for open and robotic-assisted prostatectomies were analyzed.

Results Over a 7-year period (2000-2006), a total of 1252 radical prostatectomies were performed by 17 urologists: 469 (37\%) ORPs and 783 (63\%) RARPs. The total number of prostatectomies increased annually during this
\end{abstract}

R. Munver $(\bowtie) \cdot$ I. A. Volfson · S. S. Kesler ·

I. S. Sawczuk

Department of Urology, Hackensack University Medical Center, 360 Essex Street, Suite 403, Hackensack, NJ 07601, USA

e-mail: rmunver@humed.com

R. Munver · I. S. Sawczuk

The Cancer Center, Hackensack University Medical Center, Hackensack, NJ, USA

R. Munver · M. Nazmy · I. S. Sawczuk University of Medicine and Dentistry of New Jersey, New Jersey Medical School, Newark, NJ, USA time period. The robotic-assisted procedure was predominantly performed by three (18\%) urologists from 20012003 , seven $(41 \%)$ in 2004, nine $(53 \%)$ in 2005, and 11 $(65 \%)$ in 2006 . As more urologists became trained in robotic-assisted surgery, the trend gradually shifted towards robotic-assisted prostatectomy. In 2001, only $9.6 \%$ of all radical prostatectomies at our institution were performed with robotic assistance; in 2006, this had risen to $92.8 \%$.

Conclusion The acquisition of the da Vinci ${ }^{\mathrm{TM}}$ Surgical System has allowed robotic-assisted surgery to be an available alternative to open surgery at a single institution. The implementation of robotic technology has led to the gradual adoption of robotic-assisted radical prostatectomy by many of the urologists that surgically treat prostate cancer. As a result, the percentage of open prostatectomies has steadily decreased over time, while trends in robotic-assisted prostatectomies have increased. The impact of robotics also appears to have had an influential effect on the total number of prostatectomies performed annually.

Keywords da Vinci - Practice patterns - Prostate cancer · Robotics · Telesurgery

\section{Introduction}

During the past two decades, the introduction of laparoscopic surgery has transformed the field of urology. At many institutions, laparoscopic nephrectomy is now considered the standard of care for the management of localized renal cell carcinoma. Despite the growing acceptance of laparoscopy for renal surgery in the United States, however, the implementation of laparoscopic techniques for the management of localized prostate cancer has lagged behind. 
Several factors account for the diminished lack of adoption among urologic surgeons. The complexity of a radical prostatectomy lies in the fact that the successful completion of this procedure requires skills in both extirpative and reconstructive techniques. In a laparoscopic approach, the confined space of the pelvis and limitations of available instrumentation make this operation cumbersome. The nerve-sparing portion of the procedure requires utmost precision and further adds to the difficulty level. Reconstruction of the urethrovescial anastomosis requires being comfortable in intracorporeal laparoscopic suturing. Although this technique can be mastered with adequate training and practice, the learning curve is steep and, consequently, prohibitive to many surgeons. These pitfalls, combined with a natural conservatism within the field, have slowed the implementation of laparoscopic radical prostatectomy.

The emergence of the da Vinci ${ }^{\mathrm{TM}}$ Surgical System (Intuitive Surgical, Sunnyvale, Calif.) has revolutionized the surgical approach for localized prostate cancer. Since the Food and Drug Administration (FDA) approval of robotic-assisted radical prostatectomy (RARP) in 2001, robotic-assisted surgery has been steadily gaining acceptance in the urological community. While the number of robotic-assisted procedures utilizing the da Vinci ${ }^{\mathrm{TM}}$ Surgical System is increasing among numerous surgical specialties, RARP has outpaced all others and has been performed more than any other type of robotic-assisted procedure to date.

Robotic assistance has facilitated radical prostatectomy by surgeons with minimal laparoscopic experience as well as by advanced laparoscopists. Despite the increasing enthusiasm of surgeons for robotic-assisted surgery, individual considerations need to be addressed as to the appropriate application of this novel technology.

There have been several studies that have evaluated innumerable endpoints of robotic-assisted versus open radical prostatectomy (ORP), including quality of life, oncological efficiency, continence, sexual function, blood loss, postoperative pain, hospital stay, convalescence, safety, and cost, among many others [1-3]. There are clear and unequivocal advantages of laparoscopic techniques in the management of renal tumors. However, in the realm of prostate cancer, the robotic-assisted procedure continues to be evolving, and data appear to be improving.

This manuscript examines the practice patterns at a large medical center, where both skilled open and laparoscopic urologic surgeons are present, to determine how practice patterns have evolved since the introduction of RARP.

\section{Materials and methods}

A retrospective review of the surgical database at Hackensack University Medical Center (Hackensack, NJ) was performed from January 2000 to December 2006. To this end, radical prostatectomy procedures for presumed prostate adenocarcinoma were extracted during this time period. Procedures were manually categorized into two categories: open radical prostatectomy and robotic-assisted radical prostatectomy. Attempted robotic-assisted procedures that led to conversion to an open approach were categorized in the RARP group. Procedures were further subcategorized by calendar year of performance. The number of urologic surgeons contributing to the majority of the total number of prostatectomies was recorded. Trends for total procedures, open radical prostatectomy, and robotic-assisted radical prostatectomy were derived.

Two da Vinci ${ }^{\mathrm{TM}}$ Surgical Systems were acquired in 2000 , of which one system was dedicated for training and research purposes, while the other was for clinical use. A third da Vinci ${ }^{\mathrm{TM}}$ Surgical System was acquired for clinical use in 2002, and a fourth system was acquired for training and research purposes in 2006.

Open procedures were performed via a retropubic approach. Robotic-assisted procedures were performed via a transperitoneal or extraperitoneal approach and using a 3or 4-arm da Vinci ${ }^{\mathrm{TM}}$ System, as has been described previously [4-6].

\section{Results}

In 2000, a total of 90 open radical prostatectomies were performed at our institution. Robotic-assisted radical prostatectomy was implemented in 2001. From January 2000 through to December 2006, a total of 1252 radical prostatectomies were performed by 17 urologic surgeons. During the 2001-2003 period, three (18\%) surgeons performed the majority of the RARPs; this number rose rose to seven $(41 \%)$ in 2004, nine (53\%) in 2005, and $11(65 \%)$ in 2006 (Fig. 1). Six of the seven surgeons that initially adopted RARP had received post-residency advanced training in endourology/laparoscopy $(n=3)$ or urologic oncology $(n=3)$.

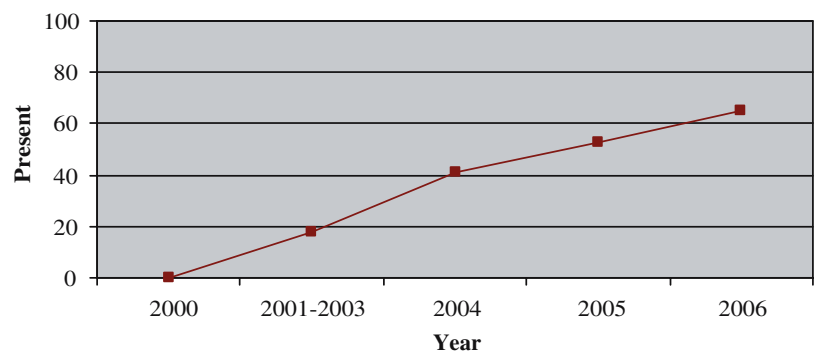

Fig. 1 Percentage of urologic surgeons performing robotic-assisted radical prostatectomy at Hackensack University Medical Center 
Of the 1252 prostatectomies, 469 (37\%) were ORPs and $783(63 \%)$ were RARPs. The total number $(n)$ of radical prostatectomies carried out annually from 2000 through to 2006 were: 90 in 2000, 125 in 2001, 143 in 2002, 151 in 2003, 143 in 2004, 265 in 2005, and 335 in 2006 (Fig. 2). The annual percentage of ORPs steadily decreased from 2001 to 2005 as follows: $90.4 \%(n=113)$ in $2001,72.0 \%$ $(n=103)$ in $2002,59.6 \%(n=90)$ in $2003,27.3 \%(n=39)$ in 2004, and $3.8 \%(n=10)$ in 2005 (Figs. 3, 4). The annual number of RARPs increased as follows: $12(9.6 \%)$ in 2001, $40(28.0 \%)$ in $2002,61(40.4 \%)$ in $2003,104(72.7 \%)$ in 2004, and 255 (96.2\%) in 2005 (Figs. 3, 4). In 2006, 311 RARPs $(92.8 \%)$ were performed.

\section{Discussion}

While open radical prostatectomy remains the gold standard for the treatment of localized prostate cancer, the adverse side effects associated with an open surgical procedure have encouraged many patients to seek less invasive options, such as external beam radiotherapy or brachytherapy. With increasing experience of urologic surgeons, this operation has made advances in the areas of oncologic efficacy, continence, and sexual function. However operative complications, blood transfusion rates, postoperative pain, hospital stay, and recovery times remain significant.

Laparoscopic radical prostatectomy was initially attempted in the early 1990s and was abandoned as a result of the technical skill that was required to perform the procedure [7]. In the late 1990s, select European centers reported that laparoscopic radical prostatectomy was feasible and offered patients the benefits of decreased postoperative pain, rapid return to normal activities, and shortened convalescence $[8,9]$. This finding was validated in the United States; however, technical aspects of oncologic control, nerve sparing, and the challenge of intracorporeal suturing led to a general consensus that the learning curve to master the procedure was steep. This issue remained prevalent over the following years as many expressed concern that the technical difficulty of this operation would limit its widespread applicability. Despite the advancement at a few academic centers, laparoscopic radical prostatectomy was not being performed by the majority of urologic oncologists or laparoscopists.

The introduction of robotic technology revived the interest of urologists in performing minimally invasive radical prostatectomy. Robotic assistance was seen as a novel alternative to conventional laparoscopic surgery, offering significant advantages over traditional open surgery. As in conventional laparoscopic procedures, robotic assistance provides patients with similar benefits associated with a minimally invasive approach.

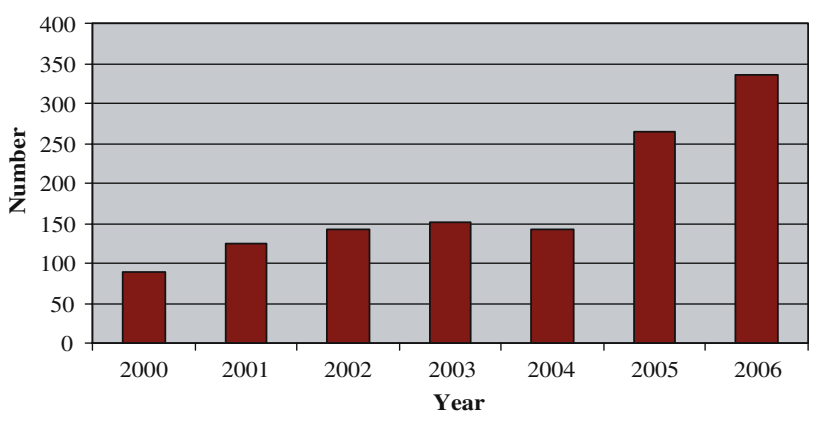

Fig. 2 Number of total radical prostatectomies performed at Hackensack University Medical Center during the period 2000-2006

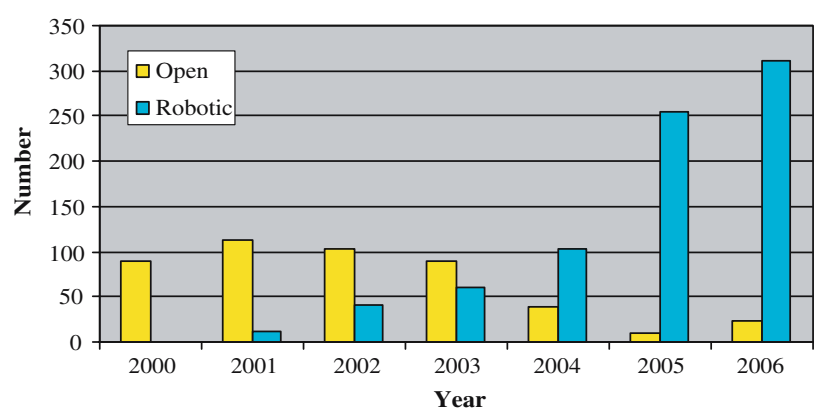

Fig. 3 Number of open and robotic-assisted radical prostatectomies performed at Hackensack University Medical Center during the period 2000-2006

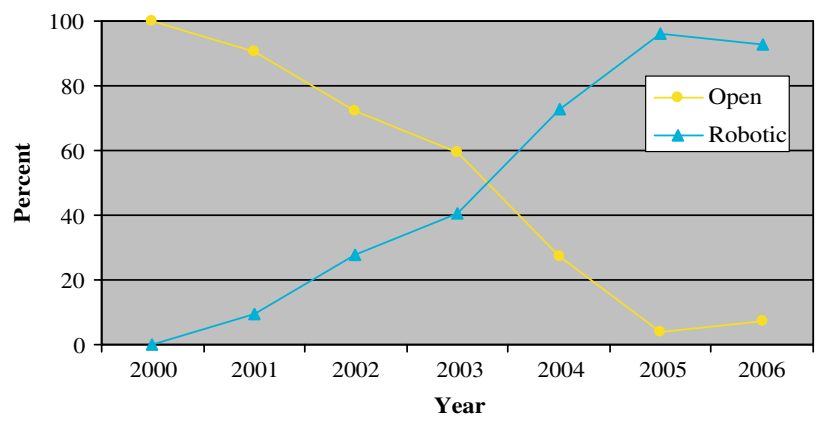

Fig. 4 Percentage of open and robotic-assisted radical prostatectomies performed at Hackensack University Medical Center during the period 2000-2006

The predominant telerobotic surgical system, the da Vinci $^{\mathrm{TM}}$ Surgical System (Intuitive Surgical), was the result of a culmination of multidisciplinary research efforts to improve upon the current foundations of minimally invasive surgery. Launched in December 1998, this robotic system was created with the intention of offering enhanced surgical capabilities, superior visualization, improved dexterity, greater precision, and ergonomic comfort. As a result of these features, a surgeon would be able to more easily perform both routine and complex laparoscopic procedures. 
Robotics has been implemented in many surgical subspecialties, however nowhere as extensively as in urology. The introduction of the da Vinci ${ }^{\mathrm{TM}}$ Surgical System and subsequent Food and Drug Administration (FDA) approval of robotic-assisted radical prostatectomy in 2001 have revolutionized the field of minimally invasive urologic surgery. In the setting of RARP, the robotic system offers several advantages over conventional laparoscopy. First, $10 \times$ optical magnification combined with a three-dimensional viewing system results in improved visualization for fine dissection and delicate maneuvering. Second, the features of motion scaling and tremor filtering allow for the more precise and less technically difficult intracorporeal suturing required during the urethrovescial anastomosis. Furthermore, EndoWrist ${ }^{\mathrm{TM}}$ technology offers 6 degrees of freedom of movement and provides an additional advantage over the 4 degrees of freedom inherent in conventional laparoscopy. This degree of precision is crucial both when dissecting the delicate neural tissue during a nerve-sparing procedure and when operating in confined areas where exaggerated movements of conventional laparoscopy can be cumbersome. Combined, these features enable the surgeon to maintain full control of these wristed laparoscopic instruments and may potentially improve surgical efficiency. An additional advantage for the surgeon is an ergonomic design of the control console, which may potentially reduce muscle strain and fatigue. Through the use of this system, non-laparoscopically trained urologic surgeons are empowered to transfer open surgical skills to the laparoscopic setting [10].

The first RARP with the da Vinci Surgical System was performed in Europe in 2000; later that year, the first of 36 RARPs was performed in the United States, a number which accounted for significantly less than $1 \%$ of all radical prostatectomies performed that year. The estimated number of RARPs performed in the United States exceeded $200(<1 \%)$ in 2001, $750(<1 \%)$ in 2002, $2500(3 \%)$ in 2003 , $8500(10 \%)$ in $2004,18,000(20 \%)$ in 2005 , and 32,000 $(35 \%)$ in 2006.

This gradual, but impressive increase can be explained by the attenuation of the steep learning curve in utilizing the robotic system. A skilled laparoscopic surgeon may be comfortable in performing a laparoscopic radical prostatectomy after approximately 40-60 cases, while a laparoscopically naive surgeon may require as many as 80-100 cases. The learning curve for RARP is also variable and is based on experience and prior training, but it is significantly better than the laparoscopic learning curve. A laparoscopically naive, yet experienced open surgeon may learn to perform RARP in as few as 8-12 cases [11, 12]. Once the learning curve is overcome, RARP appears to provide cancer control, continence, and sexual function rates that are similar to those of ORP. Additional benefits of robotic-assisted surgery for the patient lead to an improved postoperative quality of life.

The purpose of this manuscript was not to report or compare collective data on the robotic-assisted technique at our institution but to analyze the impact of robotic-technology on patients with localized prostate cancer at a large community-based hospital. Hackensack University Medical Center (HUMC) is an approximately 800-bed teaching and research hospital and is the largest provider of inpatient and outpatient services in the state of New Jersey. The hospital is the first in the United States with four da Vinci ${ }^{\mathrm{TM}}$ systems, two of which are dedicated for training and research purposes. Two systems were acquired in 2000, the year prior to FDA approval of RARP. A third system was acquired in 2002 due to a projected increase in operative volume. A fourth system was acquired in 2006 due to an increased demand for training surgical teams composed of surgeons, nurses, and technicians.

Of the initial seven urologic surgeons that performed RARP at our medical center, six had fellowship or advanced post-residency training in endourology/laparoscopy $(n=3)$ or urologic oncology $(n=3)$. Irrespective of the type of prior training, each surgeon was able to successfully utilize the da Vinci $^{\mathrm{TM}}$ Surgical System to perform robotic-assisted surgery. In 2001, the initial robotic-assisted procedures at our institution were attempted due to surgeon preference. The gradual transition to robotic-assisted radical prostatectomy arose as a result of patient/ consumer demand for a minimally invasive approach. Over a 5-year span (2001-2005), new practice patterns have evolved at HMUC as robotic-assisted radical prostatectomy has become the predominant method for surgical removal of the prostate. As is illustrated in Fig. 4, the turning point occurred in 2004, when the number of RARPs being performed surpassed the number of ORPs. Robotic-assisted procedures plateaued above the 90th percentile in 2005 . Open surgery has continued to pay a role, as certain patients elected against, or were not suitable for, a robotic approach. Furthermore, open conversion was occasionally required for reasons such as uncontrollable bleeding, inadvertent organ injury, or failure to surgically progress.

Robotic-assisted radical prostatectomy is gaining popularity in academic centers as well as in community practice [13]. At our medical center, this has translated into a near complete transition to a robotic-assisted approach within 5 years. When comparing the growth rate of RARP at Hackensack University Medical Center to that in the United States, it appears that the national rate is lagging behind by slightly more than 3 years (Fig. 5). It is difficult to predict how and when the trends for RARP in the USA will change. It is possible that the observation of $>90 \%$ saturation observed at our medical center may require more time for a similar result at the national level if adoption of 


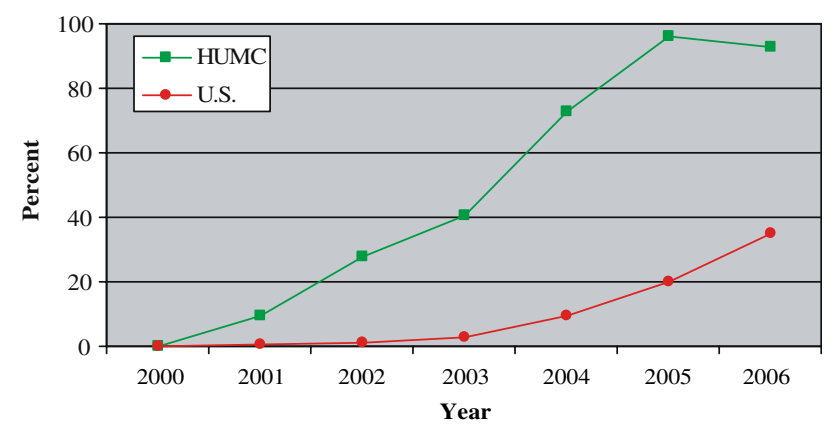

Fig. 5 Percentage of robotic-assisted radical prostatectomies performed at Hackensack University Medical Center and in the United States (2000-2006)

robotic-assisted surgery is limited by financial constraints of certain medical centers or geographic constraints of patients and physicians. Conversely, since the early learning curve for urologic surgeons performing RARP is complete, the trend for more surgeons to train and offer this procedure to their patients may result in a much more rapid dissemination of this procedure.

Robotic-assisted surgery is still in its infancy; however, centers are increasingly reporting their initial and intermediate experiences with RARP as well as with other urologic procedures [14, 15]. It is clear that the introduction of the da Vinci ${ }^{\text {TM }}$ Surgical System has had a dramatic impact on the manner in which many centers throughout the United States are approaching minimally invasive urologic surgery for localized prostate cancer. At the HUMC, the availability of robotic technology has facilitated the adoption of RARP and may be reflective of future trends at other institutions that offer robotic-assisted surgery.

\section{Conclusion}

The next generation of surgical innovation has arrived in the form of the da Vinci ${ }^{\mathrm{TM}}$ Surgical System. This technology offers a myriad of benefits to both the patient and surgeon - more specifically, a method of minimally invasive cancer control with equally minimal quality of life impacts. The expanding role of robotic-assisted surgery may facilitate radical prostatectomy, a truly complex extirpative and reconstructive procedure, especially for surgeons with minimal laparoscopic experience but also for advanced laparoscopists. The experience at our medical center is an example of the progression of the field towards minimally invasive techniques.

\section{References}

1. Tewari A, Shrivasatava A, Menon M (2003) A prospective comparison of radical retropubic and robot-assisted prostatectomy: experience in one institution. BJU Int 92:205-210

2. Menon M, Tewari A, Peabody JO et al. (2004) Vattikuti Institute prostatectomy, a technique of robotic radical prostatectomy for management of localized carcinoma of the prostate: experience of over 1100 cases. Urol Clin North Am 31:701-717

3. Ahlering TE, Woo D, Eichel L, Lee DI, Edwards R, Skarecky DW (2004) Robot-assisted versus open radical prostatectomy: a comparison of one surgeon's outcomes. Urology 63:819-822

4. Tewari A, Peabody J, Sarle R et al. (2002) Technique of da Vinci robot-assisted anatomic radical prostatectomy. Urology 60:569572

5. Sundaram CP, Koch MO, Gardner TT et al. (2005) Utility of the fourth arm to facilitate robot-assisted laparoscopic radical prostatectomy. BJU Int 95:183-186

6. Esposito MP, Ilbeigi P, Ahmed M et al. (2005) Use of fourth arm in da Vinci robot-assisted extraperitoneal laparoscopic prostatectomy: novel technique. Urology 66:649-652

7. Schuessler WW, Schulam PG, Clayman RV et al. (1997) Laparoscopic radical prostatectomy: initial short-term experience. Urology 50:854-857

8. Abbou CC, Salomon L, Hoznek A et al. (2000) Laparoscopic radical prostatectomy: preliminary results. Eur Urol 37:615620

9. Guillonneau B, Vallancien G (2000) Laparoscopic radical prostatectomy: the Montsouris technique. J Urol 163:1643-1649

10. Ahlering TE (2004) Robotic versus laparoscopic radical prostatectomy. Nat Clin Pract Urol 1:58-59

11. Ahlering TE, Skarecky D, Lee D et al. (2003) Successful transfer of open surgical skills to a laparoscopic environment using a robotic interface: initial experience with laparoscopic radical prostatectomy. J Urol 170:1738-1741

12. Reiter RE (2005) Robotically assisted laparoscopic prostatectomy: getting started. Am J Urol Rev 3:9-12

13. Patel VR, Tully AS, Holmes R et al. (2005) Robotic radical prostatectomy in the community setting - the learning curve and beyond: initial 200 cases. J Urol 174:269-272

14. Patel V (2005) Robotic-assisted laparoscopic dismembered pyeloplasty. Urology 66:45-49

15. Caruso RP, Phillips CK, Kau E et al. (2006). Robot assisted laparoscopic partial nephrectomy: initial experience. J Urol 176:36-39 